\title{
Hallazgos radiológicos habituales posteriores a cirugía bariátrica
}

\author{
Fernanda Ungson-García, ${ }^{1}$ Gilberto Ungson-Beltrán, ${ }^{2}$ \\ Carolina González-Vergara, ${ }^{3}$ Ignacio Antillón-Valenzuela ${ }^{4}$
}

\section{Resumen}

La obesidad es una enfermedad considerada epidemia mundial que constituye un importante problema de salud pública. Durante las últimas dos décadas, la cirugía bariátrica se ha convertido en el tratamiento de elección para el abordaje de la obesidad mórbida. Las técnicas quirúrgicas por laparoscopia más empleadas incluyen la derivación gástrica con reconstrucción en $Y$ de Roux, la gastrectomía vertical en manga y la derivación gástrica de una sola anastomosis. Los estudios fluoroscópicos gastrointestinales y la tomografía abdominal computada son los estudios de elección para la evaluación de pacientes postoperados de este tipo de cirugías. El objetivo principal de este artículo es presentar la anatomía quirúrgica de cada cirugía y los hallazgos radiológicos postquirúrgicos habituales esperados en individuos con un IMC $>38 \mathrm{~kg} / \mathrm{m}^{2}$ en el Hospital CIMA en Hermosillo, Sonora, México, en un periodo comprendido de enero de 2013 a enero de 2015.

Palabras clave: Cirugía bariátrica, derivación gástrica en $Y$ de Roux, Y de Roux, derivación gástrica de una sola anastomosis, gastrectomía vertical en manga, manga gástrica.

Resumen
La obesidad es una enfermedad considerada epidemia
mundial que constituye un importante problema de sa-
lud pública. Durante las últimas dos décadas, la cirugía
bariátrica se ha convertido en el tratamiento de elección
para el abordaje de la obesidad mórbida. Las técnicas
quirúrgicas por laparoscopia más empleadas incluyen la
derivación gástrica con reconstrucción en Y de Roux, la
gastrectomía vertical en manga y la derivación gástrica de
una sola anastomosis. Los estudios fluoroscópicos gas-
trointestinales y la tomografía abdominal computada son
los estudios de elección para la evaluación de pacientes
postoperados de este tipo de cirugías. El objetivo principal
de este artículo es presentar la anatomía quirúrgica de
cada cirugía y los hallazgos radiológicos postquirúrgicos
habituales esperados en individuos con un IMC > 38 kg/m²
en el Hospital CIMA en Hermosillo, Sonora, México, en un
periodo comprendido de enero de 2013 a enero de 2015 .
Palabras clave: Cirugía bariátrica, derivación gástrica en Y
de Roux, Y de Roux, derivación gástrica de una sola anas-
tomosis, gastrectomía vertical en manga, manga gástrica.

\footnotetext{
${ }^{1}$ Radiología e Imagen. Universidad La Salle. Hospital Ángeles Mocel.

${ }^{2}$ Cirujano Bariatra. Hospital CIMA Hermosillo, Sonora.

${ }^{3}$ Departamento de Radiología e Imagen. Jefe de la División de Educación Médica. Hospital Ángeles Mocel.

${ }^{4}$ Departamento de Radiología e Imagen. Hospital CIMA Hermosillo, Sonora.
}

Correspondencia:

Fernanda Ungson-García

Correo electrónico: patyungson@hotmail.com

Aceptado: 05-10-2016.

Este artículo puede ser consultado en versión completa en http:// www.medigraphic.com/actamedica
Key words: Bariatric surgery, Roux-en-Y gastric bypass,
Roux-en-Y, one anastomosis gastric bypass, vertical sleeve

Key words: Bariatric surgery, Roux-en-Y gastric bypass,
Roux-en-Y, one anastomosis gastric bypass, vertical sleeve gastrectomy, sleeve gastrectomy.

\section{Summary}

Obesity is considered a global epidemic disease worldwide. During the past two decades, bariatric surgery has become an increasingly popular form of treatment for morbid obesity. The most common laparoscopic bariatric procedures performed include Roux-en-Y gastric bypass, sleeve gastrectomy and one anastomosis gastric bypass. Fluoroscopic upper gastrointestinal examinations and abdominal computed tomography (CT) are the major imaging tests used to evaluate patients after these various forms of surgery. The purpose of this article is to present the surgical anatomy and normal imaging findings after surgery at fluoroscopic examinations mainly in patients with body mass index $(\mathrm{BMI})>38 \mathrm{~kg} / \mathrm{m}^{2}$ that underwent exclusively these surgery techniques in CIMA Hospital in Hermosillo, Sonora, Mexico, in the period from January 2013 to January 2015.

\section{INTRODUCCIÓN}

La prevalencia de la obesidad mórbida en México ha sufrido uno de los incrementos más rápidos de 1988 a 2012 respecto a las cifras reportadas a nivel mundial, con un aumento del 9.5 al 35.2\%. Según lo reportado en la última Encuesta de Salud y Nutrición, se ha documentado que el aumento sostenido de esta enfermedad es más evidente en la región norte del país, con una prevalencia de $17.1 \%$, al comparar con la Ciudad de México, con un $15.9 \%$, y el sur, con $14.9 \%{ }^{1}$

Las personas con obesidad mórbida que cambian su estilo de vida adecuando su dieta y realizando ejercicio, y se someten a terapias farmacológicas consiguen una pérdida de peso de alrededor de $10 \%$ a mediano plazo; sin embargo, la evidencia clínica indica que dicha disminución 
no es suficiente para reducir o controlar las comorbilidades asociadas a la enfermedad, como la diabetes mellitus tipo 2 , las dislipoproteinemias y la hipertensión arterial. ${ }^{2,3}$

En este contexto, es reconocido que el tratamiento más efectivo para combatir la obesidad severa es la cirugía bariátrica, que engloba tres grandes grupos: cirugías restrictivas que producen disminución de peso al limitar la ingesta (banda gástrica ajustable y gastrectomía vertical en manga), cirugías malabsortivas que llevan a la disminución de peso al interferir con los procesos de digestión y absorción (Scopinaro y cruce duodenal) y los procedimientos mixtos como derivación intestinal. ${ }^{4}$

Hasta hace algunos años, las complicaciones de estas cirugías eran tan graves como la misma obesidad; sin embargo, en la actualidad, las variaciones técnicas y el abordaje laparoscópico hacen que éstas sean menos invasivas, más seguras y eficaces.

El papel del radiólogo es determinante en la valoración del paciente postoperado de cirugía bariátrica, quien requiere pruebas de imagen como estudios fluoroscópicos especializados y tomografía computada abdominopélvica para determinar la pulcritud del procedimiento o, bien, para detectar posibles complicaciones. La creciente expansión en esta disciplina hace que cada vez sea más difícil distinguir los hallazgos habituales de cada tipo quirúrgico..$^{5,6}$

El objetivo del presente artículo es describir los hallazgos radiológicos postquirúrgicos habituales en cada una de las diferentes técnicas de cirugía que con mayor frecuencia se realizan en el Hospital CIMA Hermosillo, las cuales comprenden la derivación gástrica en Y de Roux (BPGYR), la gastrectomía vertical en manga (GVM) y la derivación gástrica de una anastomosis (BPGUA). Particularmente, y debido al aumento en la prevalencia de la obesidad en nuestro país, sobre todo en la zona norte, ${ }^{1}$ ha incrementado el número de cirugías bariátricas en el Hospital CIMA Hermosillo en el estado de Sonora, México.

\section{Derivación gástrica en Y de Roux}

La BPGYR es la intervención quirúrgica más común para el tratamiento de la obesidad severa. ${ }^{7}$ Consiste en realizar una división del estómago con grapas de titanio formando una bolsa o reservorio gástrico que va unido directamente al intestino delgado mediante una anastomosis (Figura 1A).

\section{Gastrectomía vertical en manga}

La GVM consiste en la resección de aproximadamente 75$80 \%$ del estómago, conservando la unión esofagogástrica y el píloro (Figura 1B).

\section{Derivación gástrica de una sola anastomosis}

La BPGUA es una técnica considerada variante de la BPGYR, que consiste en la formación de un reservorio gástrico y una sola anastomosis gastroyeyunal en asa (Figura 1C).8,9

\section{MATERIAL Y MÉTODO}

Durante el periodo comprendido entre enero de 2013 y enero de 2015 se recolectaron 217 casos de individuos que fueron sometidos a cirugía bariátrica en el Hospital CIMA en Hermosillo, Sonora, México. Se eligieron casos de personas con IMC > $38 \mathrm{~kg} / \mathrm{m}^{2}$ y sin antecedentes de cirugía bariátrica previa. De los 217 casos, se excluyeron 100 por no cubrir con los criterios de selección y se incluyeron 132, que se distribuyeron de la siguiente manera: 75 sujetos postoperados de gastrectomía vertical en manga (GVM), 38 postoperados de derivación gástrica de una anastomosis (BPGUA) y 19 postoperados de derivación gástrica con reconstrucción en Y de Roux (BPGYR). Todas las cirugías fueron laparoscópicas, realizadas por un solo cirujano, y a todos los pacientes se les realizó un estudio de tránsito esofagogastrointestinal con $50 \mathrm{~mL}$ de medio de
A

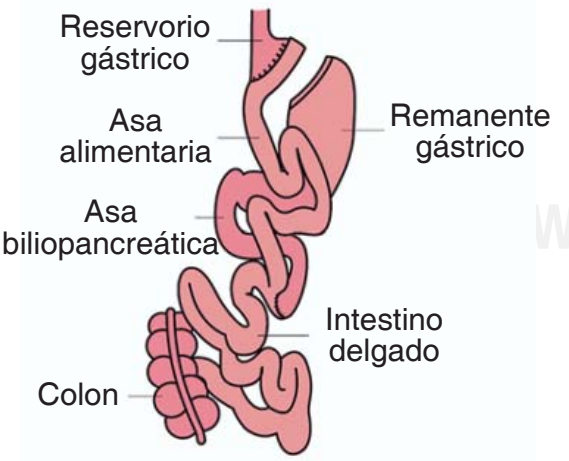

B

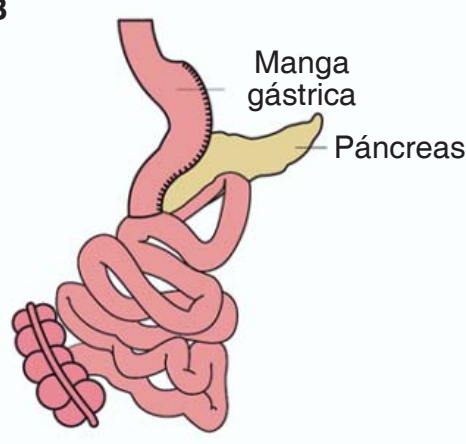

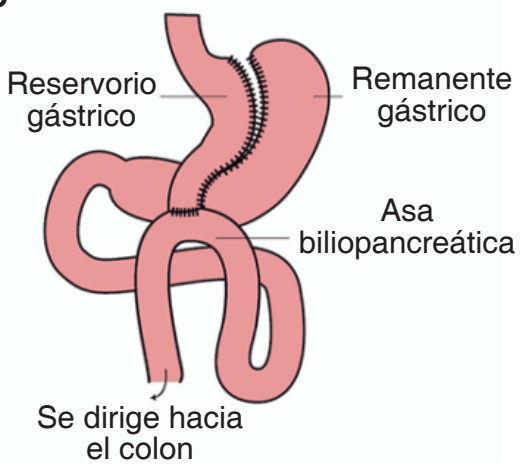

Figura 1. Esquemas de técnica de BPGYR (A), GVM (B) y BPGUA (C). 
contraste hidrosoluble con 16 g de yodo diluidos en 200 $\mathrm{mL}$ de agua vía oral entre 24 y 36 horas tras la cirugía. Las proyecciones de abdomen que se utilizaron en este estudio fueron anteroposterior y oblicua izquierda.

Todas las variables incluidas en este estudio fueron tratadas de forma independiente para los análisis descriptivos. Se realizaron medidas de tendencia central y de dispersión. Se utilizaron pruebas de T de Student para las comparaciones de las variables edad e índice de masa corporal (IMC). El programa estadístico utilizado para el análisis de los datos fue Minitab versión 16.

\section{RESULTADOS}

Los datos de edad e IMC de la muestra estudiada se presentan en el cuadro 1. Las edades comprendieron de 17 a 65 años de edad y el IMC de 28.9 a $62 \mathrm{~kg} / \mathrm{m}^{2}$. Los datos fueron categorizados de acuerdo al tipo de cirugía bariátrica y al sexo. No hubo diferencias estadísticamente significativas en la variable edad en ninguna de las técnicas quirúrgicas presentadas. Tampoco se registraron diferencias en la edad de acuerdo a la variable sexo. Cuando se analizó el IMC, observamos que los hombres sometidos a BPGYR o BPGUA presentaron un IMC significativamente mayor $(p<0.05$ ) que los pacientes sometidos a GVM. La diferencia puede explicarse debido a que en el grupo quirúrgico del hospital CIMA, los criterios de selección para este tipo de técnicas quirúrgicas son individuos con IMC $>44$ o sujetos con IMC en rango de obesidad con comorbilidades asociadas como diabetes mellitus, dislipidemia e hipertensión arterial. Cabe mencionar que la determinación del tipo de técnica quirúrgica es individualizada.

Las imágenes que se muestran corresponden a los hallazgos en estudios de fluoroscopia del tracto gastrointestinal superior en proyecciones anteroposterior y oblicua izquierda, en los que se administró medio de contraste oral hidrosoluble y fueron realizados entre 24 y 36 horas tras el evento quirúrgico (Figuras 2 a 4).

\begin{tabular}{|c|c|c|c|c|c|c|}
\hline & \multicolumn{2}{|c|}{ GVM } & \multicolumn{2}{|c|}{ BPGYR } & \multicolumn{2}{|c|}{ BPGUA } \\
\hline & $\begin{array}{c}\text { Hombres } \\
n=35(47 \%)\end{array}$ & $\begin{array}{c}\text { Mujeres } \\
n=40(53 \%)\end{array}$ & $\begin{array}{c}\text { Hombres } \\
n=7(37 \%)\end{array}$ & $\begin{array}{c}\text { Mujeres } \\
\mathrm{n}=12(63 \%)\end{array}$ & $\begin{array}{c}\text { Hombres } \\
n=18(47 \%)\end{array}$ & $\begin{array}{c}\text { Mujeres } \\
n=20(53 \%)\end{array}$ \\
\hline Edad & 32 (18-65) & $32(17-65)$ & $37(21-47)$ & $40.5(32-53)$ & $43(28-60)$ & $38.5(22-56)$ \\
\hline IMC & $42.75(40-54.2)$ & $39.2(28.9-54.2)$ & $50.3(46.9-57.8)^{*}$ & $46.25(41-50)$ & $50.65(39-62)^{*}$ & $47.05(38-61)$ \\
\hline
\end{tabular}
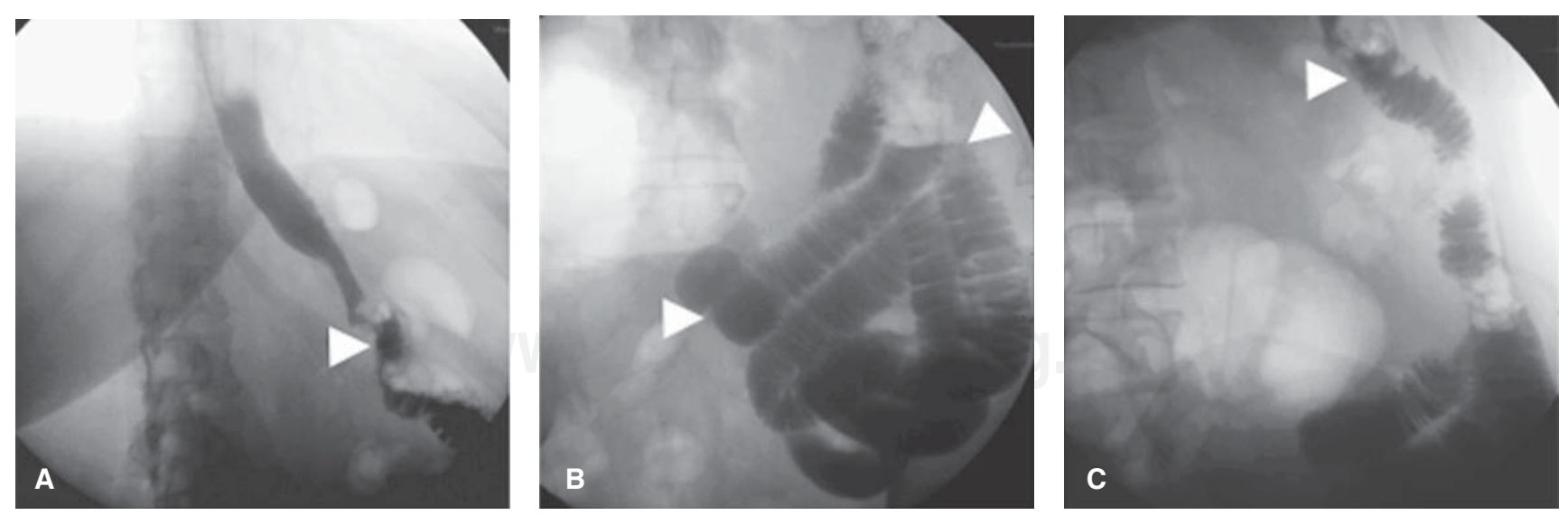

Figura 2. Anatomía postquirúrgica de BPGYR. Estudio fluoroscópico con proyecciones anteroposteriores (A y B) y oblicua izquierda (C). Se observa el reservorio gástrico (A), el asa alimentaria (C) y el intestino delgado opacificado (B). No hay datos de obstrucción del asa alimentaria. 


\section{Derivación gástrica en Y de Roux}

La anatomía postquirúrgica de BPGYR corresponde a una persona de 42 años, 24 horas después de someterse a la cirugía. Se muestra adecuado paso del medio de contraste del esófago distal hacia el reservorio gástrico, el cual tiene un volumen aproximado de $30 \mathrm{~mL}$. No hay evidencia de fuga ni comunicación entre éste y el segmento gástrico desfuncionalizado. Tampoco hay señal de fugas ni estenosis a nivel de la anastomosis gastroyeyunal. Se observa adecuado tránsito del medio hidrosoluble a través del asa alimentaria y una opacificación completa del intestino delgado distal posterior a la anastomosis yeyuno-yeyunal. Estos hallazgos descartan la presencia de obstrucción del asa alimentaria (Figura 2).

\section{Gastrectomía vertical en manga}

La anatomía postquirúrgica de GVM presentada corresponde a un paciente masculino de 51 años, 24 horas después de someterse al procedimiento. Se observa adecuado paso del medio de contraste hidrosoluble a través de la unión esofagogástrica, además de una opacificación homogénea del estómago, el cual se observa tubular, largo y delgado, sin evidencia de fugas ni torsión. Se muestra adecuado vaciamiento gástrico y paso del contraste hacia el duodeno e intestino delgado. No hay datos de fuga ni estenosis (Figura 3).

\section{Derivación gástrica de una sola anastomosis}

La anatomía postquirúrgica de BPGUA corresponde a una mujer de 38 años, 24 horas después de someterse a la ci- rugía. Se observa el paso adecuado del medio de contraste hidrosoluble del esófago distal hacia el reservorio gástrico. No hay evidencia de dilatación de éste ni de fugas del medio de contraste en el sitio de la anastomosis. Observamos la opacificación del asa eferente y aferente; esta última es esperada por reflujo retrógrado desde el reservorio gástrico, que normalmente desaparece por las ondas peristálticas. Se puede observar la opacificación del asa eferente y de asas del intestino delgado distal (Figura 4).

\section{DISCUSIÓN}

La fluoroscopia de tracto gastrointestinal alto (FTGl) y el uso de colorantes como el azul de metileno son los estudios radiológicos que con mayor frecuencia se realizan en el postquirúrgico temprano de la cirugía bariátrica, es decir entre 24 y 48 horas después de concluido el procedimiento quirúrgico. La FTGI superior aporta información detallada sobre las estructuras extraluminales y su alta resolución espacial permite evaluar tanto los cambios postquirúrgicos como las probables complicaciones que requieren atención inmediata, como las fugas del medio de contraste o la estenosis de las áreas anastomóticas. Se entiende como fuga del medio de contraste la extravasación de éste del interior del tubo digestivo hacia la cavidad abdominal, mientras que la estenosis anastomótica es un área de estrechamiento a nivel de la unión gastroyeyunal con dilatación de la bolsa gástrica y paso retrasado del medio de contraste al asa yeyunal. ${ }^{10}$

Dentro de los hallazgos radiológicos habituales en la cirugía bariátrica destacan la presencia de un estómago
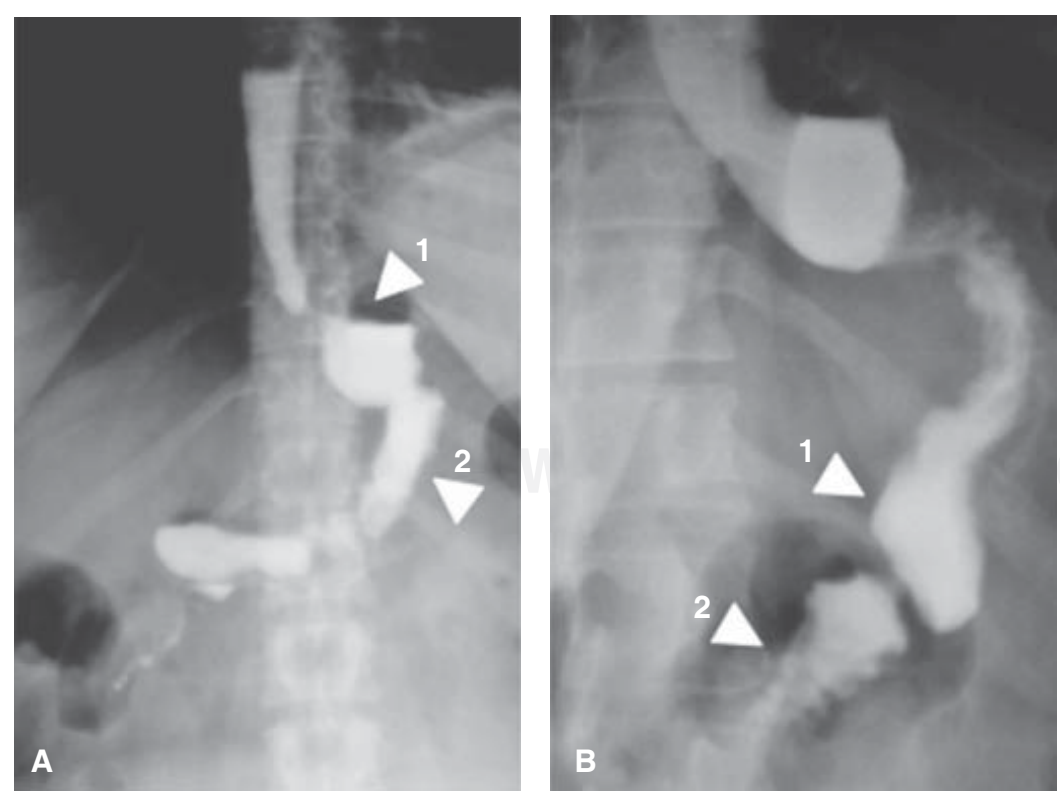

Figura 3.

Anatomía postquirúrgica de la GVM. Estudio fluoroscópico con proyecciones anteroposterior (A) y oblicua izquierda (B). Se señala la unión esofagogástrica dilatada $(\mathbf{A}, \mathbf{1})$, el estómago $(\mathbf{A}, 2)$, el duodeno $(\mathbf{B}, \mathbf{1})$ y la opacificación del intestino delgado $(\mathbf{B}, 2)$, sin evidencia de fuga del medio de contraste. 

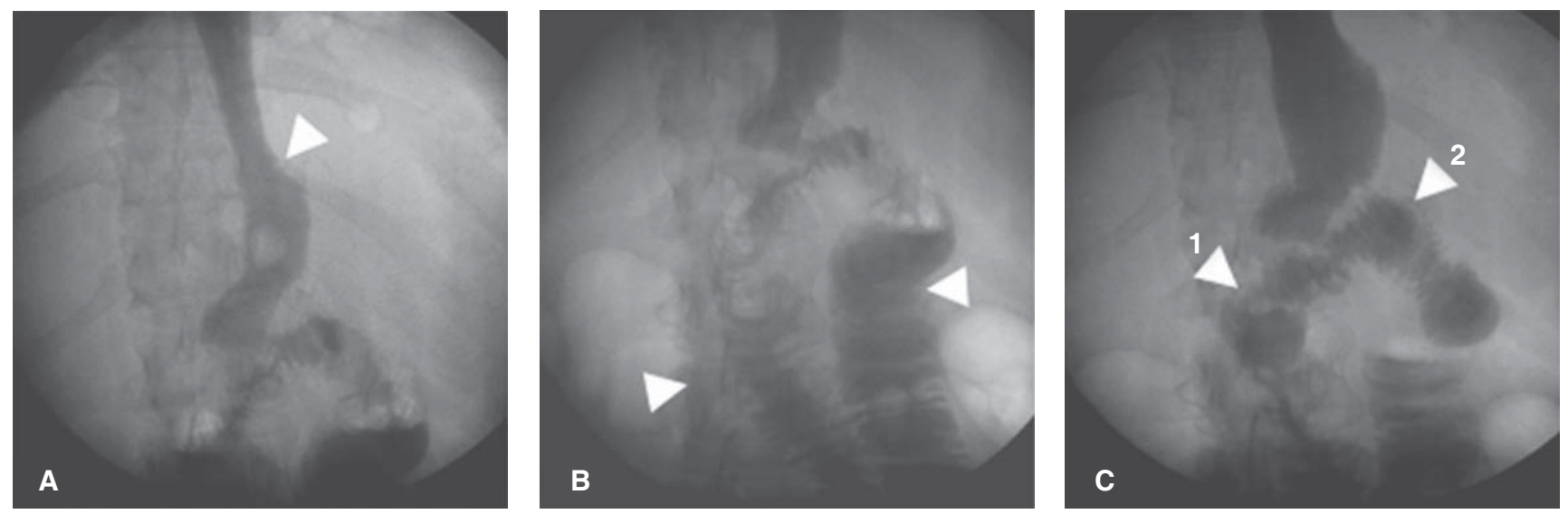

Figura 4. Anatomía postquirúrgica de BPGUA. Estudio fluoroscópico con proyecciones oblicua izquierda (A y B) y anteroposterior (C). Se observa el reservorio gástrico (A), el asa eferente $(\mathbf{C}, \mathbf{1})$, el asa aferente $(\mathbf{C}, \mathbf{2})$ y la opacificación de las asas del intestino delgado $(B)$, sin evidencia de fuga ni estenosis en la anastomosis.

tubular, largo y delgado en individuos sometidos a GVM, un reservorio gástrico con una anastomosis gastroyeyunal latero-lateral con presencia de un asa aferente y otra eferente en el caso del BPGUA y, por último, en el BPGYR, se observa un reservorio gástrico con presencia de una asa alimentaria y una anastomosis gastroyeyunal.

El objetivo principal del radiólogo es identificar las variantes "normales" postquirúrgicas de cada técnica realizada para que, a partir de ellas, logre distinguir posibles complicaciones que ameriten un segundo tiempo quirúrgico. De manera particular, la evidencia radiológica indica que las fugas a nivel de la anastomosis gastroyeyunal suelen ser las más frecuentes dentro de las complicaciones tempranas de la derivación gástrica en $\mathrm{Y}$ de Roux y la derivación gástrica de una anastomosis, mientras que en la gastrectomía vertical en manga, la principal complicación es la fuga en el extremo proximal de la línea de sutura cerca de la unión gastroesofágica o ángulo de His. ${ }^{5,11}$ Estas complicaciones son diagnosticadas en los primeros 10 días tras la cirugía. ${ }^{12}$

Las principales técnicas quirúrgicas empleadas en el Hospital CIMA, en Hermosillo, Sonora son las que se agrupan en las categorías restrictivas (GVM) y mixtas (restrictivas y malabsortivas; BPGYR y BPGUA) y se prefieren por cumplir con los objetivos establecidos por el sistema BAROS (Bariatric Analysis and Reporting Outcome System), ${ }^{13}$ los cuales son reducir el peso patológico y mantenerlo en el tiempo, curar o mejorar las comorbilidades que reducen la vida del obeso mórbido para que sus expectativas de vida sean las mismas que las de las personas de su misma edad, aportar una correcta calidad de vida (especialmente en lo que a prácticas nutricionales se refiere) e inducir un número mínimo de secuelas.

\section{Derivación gástrica en Y de Roux}

En los estudios de fluoroscopia de control posterior a la cirugía, el reservorio gástrico se observa como una pequeña estructura con volumen de aproximadamente $30 \mathrm{~mL}$, en tanto que la anastomosis gastroyeyunal aparece entre el reservorio gástrico y el yeyuno, y es una unión del extremo distal del yeyuno (previamente llevado al cuadrante superior izquierdo) con la pared gástrica por una sutura mecánica. ${ }^{5,7}$ En ausencia de complicaciones, el medio de contraste hidrosoluble debe transitar libremente hacia el asa alimentaria. El intestino delgado, por su parte, deberá mostrarse opaco. Es importante seguir el paso del medio de contraste desde el esófago hacia el interior del reservorio gástrico y hasta la anastomosis gastroyeyunal para descartar posibles fugas o estenosis.

\section{Gastrectomía vertical en manga}

En los estudios fluoroscópicos postquirúrgicos, habitualmente se observa una estructura tubular, larga y delgada. Es importante detectar la presencia de zonas del estómago que pudieran haber sido resecadas de forma insuficiente, especialmente en el fundus y el antro gástrico, los cuales son fácilmente observados en proyecciones oblicuas. La literatura concuerda en señalar que el radiólogo deberá realizar una valoración del tránsito del material de contraste por todo el trayecto de la manga gástrica para verificar el paso y el vaciamiento gástrico a través del píloro. Para que los hallazgos radiológicos cumplan con el criterio de "habituales", deberá descartarse la presencia de fugas del material de contraste, así como estenosis. ${ }^{14,15}$ En la gastrectomía vertical en manga, el sitio más frecuente de 
fuga del medio de contraste es el ángulo de His, y el de estenosis es la incisura angularis. La fuga en el ángulo de His generalmente se asocia a una estenosis distal, que ocasiona el aumento de presión en el interior del estómago, con la consecuente disrupción de la línea de grapas. La estenosis en la incisura angularis, por su parte, se asocia a diferentes causas, entre las que destacan edema de la mucosa gástrica, compresiones extrínsecas y/o a angulación muy aguda a nivel de la incisura angularis, denominada "kinking" en la literatura universal. 9,15

\section{Derivación gástrica de una sola anastomosis}

Los cambios radiológicos esperados constan de un reservorio gástrico más alargado que el habitual y una anastomosis latero-lateral izquierda y distal. En esta técnica es fundamental que el radiólogo evalúe la longitud del reservorio gástrico y el paso del medio de contraste a través de la anastomosis para descartar fugas o estenosis. A través de la anastomosis se deberá observar el paso de material de contraste hacia el asa aferente por reflujo retrógrado, que será barrido por el propio peristaltismo hacia el asa eferente, lo cual provoca la opacificación de las dos asas. ${ }^{11,16}$

\section{CONCLUSIONES}

El monitoreo postquirúrgico en el sujeto que ha sido sometido a algún tipo de cirugía bariátrica es responsabilidad de un grupo interdisciplinario y exige que el radiólogo involucrado tenga conocimiento y experiencia tanto de las técnicas quirúrgicas como del manejo de los estudios del tracto gastrointestinal en sus diversas modalidades para identificar complicaciones y corregirlas en el menor tiempo posible, ya que la mayoría de éstas son predecibles, prevenibles y tratables.

\section{REFERENCIAS}

1. Gutiérrez J, Rivera-Dommarco J, Shamah-Levy T, VillalpandoHernández S, Franco A, Cuevas-Nasu L et al. Encuesta Nacional de
Salud y Nutrición 2012. Resultados Nacionales. Cuernavaca, México: Instituto Nacional de Salud Pública (MX); 2012.

2. González-Montaño V, Marín-Antúnez A, Nogales-Montero J, Zamorano-Pozo T, Moreno-Puertas A, Mora-Encinas J. Cirugía bariátrica. Lo que el radiólogo debe saber [Internet]. EPOS. 2012 [Consultado el 9 de agosto de 2015]. Disponible en: http://dx.doi. org/10.1594/seram2012/S-1392

3. González-González J, Sanz-Álvarez L, García-Bernardo C. La obesidad en la historia de la cirugía. Cir Esp. 2008; 84 (4): 188-195.

4. Solís-Ayala E, Carrillo-Ocampo L, Canché-Arenas A, Cortázar-Benítez L, Cabrera-Jardines R, Rodríguez-Weber F et al. Cirugía bariátrica: resultados metabólicos y complicaciones. Med Int Mex. 2013; 29 (5): 487-494.

5. Levine MS, Carucci LR. Imaging of bariatric surgery: normal anatomy and postoperative complications. Radiology. 2014; 270 (2): 327-341.

6. Kurian M. Imaging studies after bariatric surgery [Internet]. UpToDate. com. 2015 [cited 9 November 2015]. Available from: http://www. uptodate.com/contents/imaging-studies-after-bariatric-surgery

7. Caracela-Zeballos C, Diéguez-Tapias S, Cereceda-Pérez C, PintoVarela J. Bypass gástrico laparoscópico: cambios postoperatorios habituales y aspecto radiológico de sus complicaciones en la tomografía computarizada. Radiología. 2014; 56 (5): 413-419.

8. Chakhtoura G, Zinzindohoué F, Ghanem Y, Ruseykin I, Dutranoy JC, Chevallier JM. Primary results of laparoscopic mini-gastric bypass in a French obesity-surgery specialized university hospital. Obes Surg. 2008; 18 (9): 1130-1133.

9. Choi S, Kasama K. Bariatric and metabolic surgery. Springer Berlin Heidelberg; 2014.

10. Salazar C, Vergnaud JP, Lopera C, Uribe AL. Estenosis de la anastomosis gastroyeyunal como complicación de la derivación gástrica en cirugía bariátrica por laparoscopia: Reporte de tres casos y su manejo. Rev Colomb Cir. 2005; 20 (1): 54-58.

11. Chevallier JM, Arman GA, Guenzi M, Rau C, Bruzzi M, Beaupel N et al. One thousand single anastomosis (omega loop) gastric bypasses to treat morbid obesity in a 7-year period: outcomes show few complications and good efficacy. Obes Surg. 2015; 25 (6): 951-958.

12. Luber SD, Fischer DR, Venkat A. Care of the bariatric surgery patient in the emergency department. J Emerg Med. 2008; 34 (1): 13-20.

13. Larrad A, Sánchez-Cabezudo C. Indicadores de calidad en cirugía bariátrica y criterios de éxito a largo plazo. Cir Esp. 2004; 75 (5): 301-304.

14. Márquez MF, Ayza MF, Lozano RB, Morales-M del M, Díez JM, Poujoulet RB. Gastric leak after laparoscopic sleeve gastrectomy. Obes Surg. 2010; 20 (9): 1306-1311.

15. Zundel N, Hernandez JD, Galvao-Neto M, Campos J. Strictures after laparoscopic sleeve gastrectomy. Surg Laparosc Endosc Percutan Tech. 2010; 20 (3): 154-158.

16. Carbajo-Caballero M, García-Caballero M, Ortiz de Solórzano J, Osorio D, García-Lanza C. Bypass gástrico laparoscópico-robótico de una sola anastomosis: resultados preliminares sobre 300 casos. Rev Mex Cir Endoscop. 2005; 6 (1): 5-10. 